This is a self-archived version of an original article. This version may differ from the original in pagination and typographic details.

Author(s): Salonen, Pasi; Peuronen, Anssi; Sinkkonen, Jari; Lehtonen, Ari

Title: Oxidovanadium(v) complexes with I-proline-based amino acid phenolates

Year: 2019

Version: Accepted version (Final draft)

Copyright: (c) 2019 Elsevier B.V.

Rights: $C C B Y-N C-N D 4.0$

Rights url: https://creativecommons.org/licenses/by-nc-nd/4.0/

Please cite the original version:

Salonen, P., Peuronen, A., Sinkkonen, J., \& Lehtonen, A. (2019). Oxidovanadium(v) complexes with I-proline-based amino acid phenolates. Inorganica Chimica Acta, 489, 108-114.

https://doi.org/10.1016/j.ica.2019.02.011 


\title{
Oxidovanadium(v) complexes with L-proline-based amino acid phenolates
}

Pasi Salonena, Anssi Peuronen ${ }^{\mathrm{a}, \mathrm{b}}$, Jari Sinkkonenc, Ari Lehtonen ${ }^{\mathrm{a}}$

a) Group of Inorganic Materials Chemistry, Department of Chemistry, University of Turku, FI-20014, Turku, Finland

b) Laboratory of Inorganic Chemistry, Department of Chemistry, University of Jyväskylä, FI-40014, Jyväskylä, Finland

c) Instrument Center, University of Turku, FI-20014, Turku, Finland

\author{
*Ari Lehtonen \\ Department of Chemistry, Group of Inorganic Materials Chemistry \\ University of Turku \\ FI-20014, Turku, Finland \\ E-mail: ari.lehtonen@utu.fi
}

L-proline was used to prepare chiral, tridentate amino acid phenol proligands $\mathrm{H}_{2} \mathrm{~L}^{1-4}$. These proligands react with vanadium precursors $\mathrm{VO}(\mathrm{acac})_{2}, \mathrm{VOSO}_{4} \cdot 5 \mathrm{H}_{2} \mathrm{O}$ and $\mathrm{VO}(\mathrm{OPr})_{3}$ in methanol to form the corresponding oxidoalkoxidovanadium(v) complexes 1-4. The complexes crystallize from methanol, and are octahedrally coordinated with a general formula $\left[\mathrm{VO}\left(\mathrm{L}^{1-4}\right)(\mathrm{OMe})(\mathrm{MeOH})\right]$. In solution, however, they adopt several different conformations or isomeric structures depending on the solvent.

\section{Highlights}

- Tridentate amino acid phenol proligands were made of L-proline.

- Proligands react with various vanadium precursors to form oxidoalkoxidovanadium(v) complexes.

- In solution, the complexes adopt different conformations or isomeric structures.

- The solid-state structures resemble the active site found in vanadium bromoperoxidase. 


\section{Introduction}

The coordination chemistry of vanadium is generally studied due to the biological relevance and catalytic properties of different vanadium-based systems.[1-3] Especially, bioinorganic chemistry of vanadium, i.e. the central role of vanadium in certain haloperoxidases (VHPOs) and nitrogenase enzymes as well as the insulin-like effects of vanadium compounds have motivated synthetic chemists to prepare model compounds for structural studies and reactivity tests.[4] For example, the active sites of VHPOs have been modelled by vanadium phenoxides and catecholates. There are also some examples on the catechol oxidase mimicking activity of molecular vanadium(Iv) and vanadium(v) complexes.[5-9] Moreover, artificial oxidovanadium(v) complexes can be used as catalysts in a number of organic oxidation reactions, e.g. sulfoxidation, epoxidation and oxidative bromination.[10-13] To date, some of the most active reported oxidovanadium(v) complexes for epoxidation, sulfoxidation and oxidative halogenation - that model vanadium haloperoxidases structurally and functionally - are based on tetradentate atrane-like aminotrisphenolate ligands.[14,15]

In synthetic coordination chemistry, the environment of the metal centre can be modified by using diverse ligands. The different electronic and steric properties of ligands may have a remarkable effect on the reactivity of the metal species. In this regard, aminophenolates, and aminobisphenolates in particular, are a large family of well-known multidentate ligands that have found extensive use in the coordination chemistry of most transition metals. [16] This is because they are relatively easy to synthesize and as such they are easily modified to offer different steric and electronic properties. Although many complexes based on these ligands exist, aminophenols with amino acid side arm coupled to phenols, or amino acid phenols, are scarcely reported. These kinds of amino acid-based ligands can better mimic natural biomolecules, which comprise most enzyme active sites, including vanadium haloperoxidases (figure 1). 

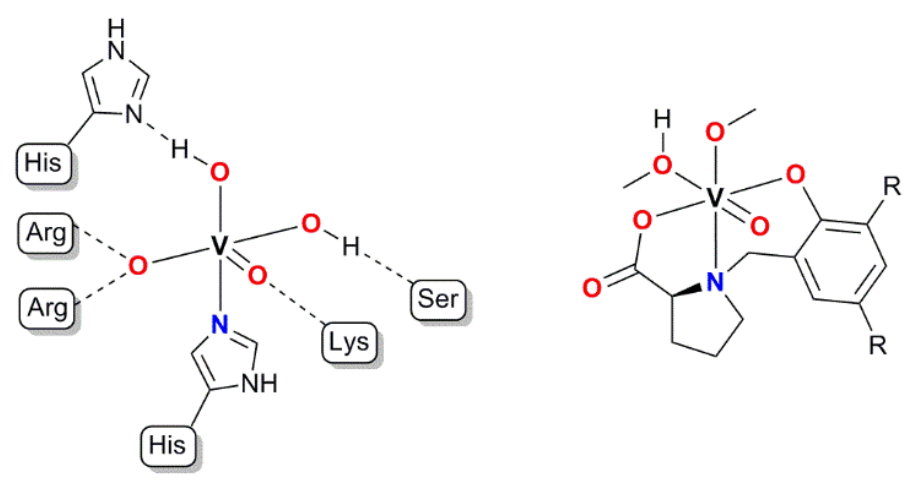

Figure 1. The oxidovanadium(v) centre of vanadium-depended bromoperoxidase as found in brown seaweed Ascophyllum nodosum (left).[17][18] General structure of the oxidoalkoxidovanadium(v) complexes prepared in this study (right).

To this end, in the present work, we have used the amino acid phenol derivatives of L-proline as ligands to introduce a biomimetic environment around the oxidovanadium(v) centre. Herein we describe the preparation and characterization of four oxidoalkoxidovanadium(v) complexes derived from disubstituted (2-hydroxyphenol)-L-prolines.

\section{Experimental}

All syntheses and manipulations were performed under ambient conditions. All solvents were of analytical grade unless stated otherwise and were used as received. All chemicals used in syntheses or catalysis were from commercial sources, of reagent grade unless stated otherwise, and used as received.

IR spectra were recorded using a Bruker VERTEX 71 FTIR spectrometer equipped with an RTDLaDTGS detector. 32 scans were performed in ATR mode for each individual measurement with the Harrick VideoMVP accessory, in which the sample is compressed against a diamond. UV-Vis and CD spectra were recorded in $\mathrm{MeOH}, \mathrm{MeCN}$ or $\mathrm{CHCl}_{3}$ using a $\emptyset 1 \mathrm{~cm}$ quartz cuvette with an Agilent CARY60 or a Chirascan qCD spectrometer. High-resolution mass spectra were recorded with a Bruker Daltonics MicrOTOF-Q II electrospray ionization time-of-flight mass spectrometer in both negative and positive ionization mode. For a complete list of IR, UV-Vis, $\mathrm{CD}$ and mass spectra and data, refer to the electronic supplementary information.

\subsection{Syntheses}


(3,5-di-tert-butyl-2-hydroxybenzyl)-L-proline, $\left(\mathbf{H}_{2} \mathbf{L}^{1}\right)$. This proligand was synthesized following a modified published procedure.[19] $20 \mathrm{mmol}$ 2,4-di-tert-butylphenol (4.13 g), 20 $\mathrm{mmol}$ paraformaldehyde $(0.60 \mathrm{~g})$ and $20 \mathrm{mmol}$ L-proline $(2.30 \mathrm{~g})$ were suspended in $30 \mathrm{ml}$ technical methanol in a $100 \mathrm{ml}$ round-bottomed flask equipped with a magnetic stir-bar and a reflux condenser. The reaction mixture was stirred, heated to boil and refluxed for 16 hours. The target compound was obtained as a slightly purple powder after purification via flash chromatography using $\mathrm{CH}_{2} \mathrm{Cl}_{2}$ : $\mathrm{MeOH}$ (ca. 95:5 V:V) eluent mixture. Proligands $\mathrm{H}_{2} \mathrm{~L}^{2-4}$ were synthesized analogously. See electronic supplementary information for more details. Yield: 5.31 g (80 \%). M.p. ca. $160{ }^{\circ} \mathrm{C}$ (decomposes). ${ }^{1} \mathrm{H}$ NMR $\left(\mathrm{CDCl}_{3}, 400 \mathrm{MHz}, \mathrm{TMS}\right) \delta 9.01$ (s, 2H), $7.31(\mathrm{~d}, 1 \mathrm{H}, \mathrm{J}=2.2 \mathrm{~Hz}), 6.92(\mathrm{~d}, 1 \mathrm{H}, \mathrm{J}=2.2 \mathrm{~Hz}), 4.62(\mathrm{~d}, 1 \mathrm{H}, \mathrm{J}=13 \mathrm{~Hz}), 3.90(\mathrm{~d}, 1 \mathrm{H}, \mathrm{J}=13 \mathrm{~Hz})$, $3.79(\mathrm{t}, 1 \mathrm{H}, \mathrm{J}=7.0 \mathrm{~Hz}), 3.41(\mathrm{~m}, 1 \mathrm{H}), 2.79(\mathrm{q}, 1 \mathrm{H}, \mathrm{J}=10 \mathrm{~Hz}), 2.30(\mathrm{~m}, 2 \mathrm{H}), 1.95(\mathrm{~m}, 2 \mathrm{H}), 1.41(\mathrm{~s}$,

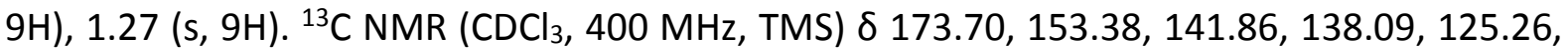
$124.81,120.12,67.62,56.84,53.06,35.03,34.16,31.59,29.86,28.73,23.01 . \mathrm{IR}\left(\mathrm{KBr}, \mathrm{cm}^{-1}\right)$ : 2952s, 2905m, 2866m, 1709s, 1611s, 1481s, 1445s, 1361s, 1302s, 1230vs, 1201vs, 876m, $725 \mathrm{~m}, 689 \mathrm{~m}, 649 \mathrm{~m}$. UV-Vis (MeCN; $\left.\lambda_{\max } / \mathrm{nm}\left(\varepsilon / \mathrm{M}^{-1} \mathrm{~cm}^{-1}\right)\right) 283(2660)$.

(3-tert-butyl-2-hydroxy-5-methylbenzyl)-L-proline, $\left(\mathbf{H}_{2} \mathrm{~L}^{2}\right)$. Yield: 4.07 g (70 \%). M.p. ca. 135 ${ }^{\circ} \mathrm{C}$ (decomposes). ${ }^{1} \mathrm{H}$ NMR ( $\left.\mathrm{CDCl}_{3}, 400 \mathrm{MHz}, \mathrm{TMS}\right) \delta 7.97$ (s, 2H), 7.06 (d, 1H, J = $1.5 \mathrm{~Hz}$ ), 6.72 (d, $1 \mathrm{H}, \mathrm{J}=1.5 \mathrm{~Hz}), 4.53(\mathrm{~d}, 1 \mathrm{H}, \mathrm{J}=13 \mathrm{~Hz}), 3.82(\mathrm{~d}, 1 \mathrm{H}, \mathrm{J}=13 \mathrm{~Hz}), 3.74(\mathrm{t}, 1 \mathrm{H}, \mathrm{J}=7.3 \mathrm{~Hz}$ ) 3.38 $(\mathrm{m}, 1 \mathrm{H}), 2.76(\mathrm{~m}, 2 \mathrm{H}), 2.22(\mathrm{~s}, 3 \mathrm{H}), 1.93(\mathrm{~m}, 2 \mathrm{H}), 1.38(\mathrm{~s}, 9 \mathrm{H}) .{ }^{13} \mathrm{C} \mathrm{NMR}\left(\mathrm{CDCl}_{3}, 400 \mathrm{MHz}, \mathrm{TMS}\right)$ $\delta 173.73,153.51,138.67,128.91,128.54,128.45,120.74,67.52,56.55,53.15,34.71,29.77$, 28.75, 23.05, 20.73. IR (KBr, cm $\left.{ }^{-1}\right)$ 2949s, 2915m, 2867m, 1712m, 1623s, 1481s, 1444s, 1390s, 1359s, 1298s, 1241s, 1203s, 862s, 762s, 688s, 588m, 528m. UV-Vis (MeCN; $\lambda_{\max } / \mathrm{nm}\left(\varepsilon / \mathrm{M}^{-1}\right.$ $\left.\left.\mathrm{cm}^{-1}\right)\right) 283(2060)$.

(5-tert-butyl-2-hydroxy-3-methylbenzyl)-L-proline, $\left(\mathbf{H}_{2} \mathrm{~L}^{3}\right)$. Yield: $3.50 \mathrm{~g}(60$ \%). M.p. ca. 145$160{ }^{\circ} \mathrm{C}$ (decomposes). ${ }^{1} \mathrm{H}$ NMR $\left(\mathrm{CDCl}_{3}, 400 \mathrm{MHz}, \mathrm{TMS}\right) \delta 7.97(\mathrm{~s}, 2 \mathrm{H}), 7.06$ (d, $\left.1 \mathrm{H}, \mathrm{J}=1.5 \mathrm{~Hz}\right)$, $6.72(\mathrm{~d}, 1 \mathrm{H}, \mathrm{J}=1.5 \mathrm{~Hz}), 4.53(\mathrm{~d}, 1 \mathrm{H}, \mathrm{J}=13 \mathrm{~Hz}), 3.82(\mathrm{~d}, 1 \mathrm{H}, \mathrm{J}=13 \mathrm{~Hz}), 3.74(\mathrm{t}, 1 \mathrm{H}, \mathrm{J}=7.3 \mathrm{~Hz})$, $3.38(\mathrm{~m}, 1 \mathrm{H}), 2.76(\mathrm{~m}, 2 \mathrm{H}), 2.22(\mathrm{~s}, 3 \mathrm{H}), 1.93(\mathrm{~m}, 2 \mathrm{H}), 1.38(\mathrm{~s}, 9 \mathrm{H}) .{ }^{13} \mathrm{C} \mathrm{NMR}_{(\mathrm{CDCl}}, 400 \mathrm{MHz}$, TMS) $\delta$ 173.73, 153.51, 138.67, 128.91, 128.54, 128.45, 120.74, 67.52, 56.55, 53.15, 34.71, $29.77,28.75,23.05,20.73$. IR (KBr, cm $\left.{ }^{-1}\right) 2949 \mathrm{~s}, 2915 \mathrm{~m}, 2867 \mathrm{~m}, 1712 \mathrm{~m}, 1623 \mathrm{~s}, 1481 \mathrm{~s}, 1444 \mathrm{~s}$, 
1390s, 1359s, 1298s, 1241s, 1203s, 862s, 762s, 688s, 588m, 528m. UV-Vis (MeCN; $\lambda_{\max } / \mathrm{nm}$ $\left.\left(\varepsilon / \mathrm{M}^{-1} \mathrm{~cm}^{-1}\right)\right) 283(2060)$.

(2-hydroxy-3,5-dimethylbenzyl)-L-proline, $\left(\mathbf{H}_{2} \mathrm{~L}^{4}\right)$. Yield: 4.71 g $(94 \%)$. M.p. ca. $145^{\circ} \mathrm{C}$ (decomposes). ${ }^{1} \mathrm{H} \mathrm{NMR}\left(\mathrm{CDCl}_{3}, 500 \mathrm{MHz}, \mathrm{TMS}\right) \delta 8.77(\mathrm{~s}, 2 \mathrm{H}), 6.91$ (d, $1 \mathrm{H}, \mathrm{J}=1.2 \mathrm{~Hz}$ ), 6.70 (d, $1 \mathrm{H}, \mathrm{J}=1.2 \mathrm{~Hz}), 4.41(\mathrm{~d}, 1 \mathrm{H}, \mathrm{J}=13 \mathrm{~Hz}), 3.92(\mathrm{~d}, 1 \mathrm{H}, \mathrm{J}=13 \mathrm{~Hz}), 3.86(\mathrm{t}, 1 \mathrm{H}, \mathrm{J}=6.7 \mathrm{~Hz}), 3.45(\mathrm{~m}$, 1H), $2.82\left(\mathrm{q}, 1 \mathrm{H}, \mathrm{J}=8.8 \mathrm{~Hz}\right.$ ), $2.29(\mathrm{~m}, 2 \mathrm{H}), 2.23(\mathrm{~s}, 3 \mathrm{H}), 2.18(\mathrm{~s}, 3 \mathrm{H}), 1.92(\mathrm{~m}, 2 \mathrm{H}) .{ }^{13} \mathrm{C} N M R$ $\left(\mathrm{CDCl}_{3}, 500 \mathrm{MHz}\right.$, TMS) $\delta 172.64,152.81,132.84,128.65,128.55,126.39,118.38,68.05,56.27$, 53.38, 28.90, 23.31, 20.32, 16.46. IR (KBr, cm $\left.{ }^{-1}\right) 2952 \mathrm{~m}, 2917 \mathrm{~m}, 2869 \mathrm{~m}, 1709 \mathrm{~m}, 1629 \mathrm{~s}, 1490 \mathrm{~s}$, 1387s, 1356s, 1304s, 1221vs, 1157s, 1040m, 1007m, 861s, 744m, 682m, 567m. UV-Vis (MeCN; $\left.\lambda_{\max } / \mathrm{nm}\left(\varepsilon / \mathrm{M}^{-1} \mathrm{~cm}^{-1}\right)\right) 285(2500)$.

Complexes 1-4 were prepared following identical procedures, although X-ray quality crystals were obtained only for $\mathbf{1}$ and $\mathbf{4}$. The syntheses were repeated several times, and the reported values represent the average yield for each complex. Different vanadium precursors, i.e. VOSO $_{4} \cdot 5 \mathrm{H}_{2} \mathrm{O}, \mathrm{VO}(\text { acac })_{2}$ or $\mathrm{VO}(\mathrm{OPr})_{3}$ gave practically similar results. If $\mathrm{VOSO}_{4} \cdot 5 \mathrm{H}_{2} \mathrm{O}$ was used, then two equivalents of triethylamine was added as well.

[VO( $\left.\left.\mathrm{L}^{1}\right)(\mathrm{OMe})(\mathrm{MeOH})\right], 1.1 \mathrm{mmol}$ of vanadium precursor and $1 \mathrm{mmol} \mathrm{H}_{2} \mathrm{~L}^{1}$ were dissolved in $15 \mathrm{ml}$ methanol, heated to ca. $60{ }^{\circ} \mathrm{C}$ and stirred for two hours in an open $50 \mathrm{ml}$ roundbottomed flask. Brown crystals suitable for single crystal x-ray diffraction were obtained in two to three days at $-25^{\circ} \mathrm{C}$ in methanol. Yield: $0.15 \mathrm{~g}(33 \%){ }^{1} \mathrm{H}$ NMR $(\mathrm{MeOH}-d 4,500 \mathrm{MHz}$, TMS) $\delta 7.44(\mathrm{~d}, 1 \mathrm{H}, \mathrm{J}=2.3 \mathrm{~Hz}), 7.17(\mathrm{~d}, 1 \mathrm{H}, \mathrm{J}=2.3 \mathrm{~Hz}), 4,05(\mathrm{dd}, 1 \mathrm{H}, \mathrm{J} 1=8.5 \mathrm{~Hz}, \mathrm{~J} 2=2.3 \mathrm{~Hz})$, $3.81(\mathrm{~d}, 1 \mathrm{H}, \mathrm{J}=12 \mathrm{~Hz}), 3.53(\mathrm{~d}, 1 \mathrm{H}, \mathrm{J}=12.0 \mathrm{~Hz}), 3.37(\mathrm{~m}, 1 \mathrm{H}), 3.21(\mathrm{~m}, 1 \mathrm{H}), 2.39(\mathrm{~m}, 2 \mathrm{H}), 2.06$ (m, 1H), $1.89(\mathrm{~m}, 1 \mathrm{H}), 1.50(\mathrm{~s}, 9 \mathrm{H}), 1.34(\mathrm{~s}, 9 \mathrm{H}) .{ }^{13} \mathrm{C} \mathrm{NMR}$ (MeOH-d4, $\left.500 \mathrm{MHz}, \mathrm{TMS}\right) \delta$ 181.27, $163.84,143.20,134.72,123.90,123.87,123.79,72.34,57.47,56.18,48.47,34.80,33.89$, 30.75, 29.61, 26.14, 21.32. ${ }^{51} \mathrm{~V}$ NMR (MeOH-d4, $500 \mathrm{MHz}, \mathrm{VOCl}_{3}$ ) $\delta-553.73,-493.35,-466.36$. IR (KBr, cm $\left.{ }^{-1}\right) ~ 3400 w b, 2954 s, 1668 s, 1617 m, 1473 m, 1441 \mathrm{~m}, 1360 \mathrm{~m}, 1236 \mathrm{~s}, 1125 \mathrm{~s}, 1050 \mathrm{vs}$, 976s (V=O), 845s, 754s, 581vs, 539s, 484s. UV-Vis (MeCN; $\left.\lambda_{\max } / \mathrm{nm}\left(\varepsilon / \mathrm{M}^{-1} \mathrm{~cm}^{-1}\right)\right) 257(8750)$, 313 (6010), 353 (6440), 637 (1440). ESI-MS : [VO(L $\left.\left.{ }^{1}\right)(\mathrm{OMe})+\mathrm{Na}\right]^{+} \mathrm{m} / \mathrm{z}_{\text {calcd. }}=452.161, \mathrm{~m} / \mathrm{z}_{\text {found }}$. $=452.168$. ESI-MS $:\left[\mathrm{V}(\mathrm{O})_{2}\left(\mathrm{~L}^{1}\right)\right]^{-} \mathrm{m} / \mathrm{z}_{\text {calcd. }}=414.149, \mathrm{~m} / \mathrm{z}_{\text {found. }}=414.133$. Black single crystals suitable for X-ray diffraction of the dimeric 1' were obtained from moist acetonitrile at -25 ${ }^{\circ} \mathrm{C}$. 
[VO(L $\left.\left.{ }^{2}\right)(\mathrm{OMe})(\mathrm{MeOH})\right]$, 2. Yield: $0.20 \mathrm{~g}(52 \%) .{ }^{1} \mathrm{H} \mathrm{NMR}(\mathrm{MeOH}-d 4,400 \mathrm{MHz}, \mathrm{TMS}) \delta 7.20$ (d, $1 \mathrm{H}, \mathrm{J}=1.9 \mathrm{~Hz}), 6.96(\mathrm{~d}, 1 \mathrm{H}, \mathrm{J}=1.9 \mathrm{~Hz}), 4.05(\mathrm{dd}, 1 \mathrm{H}, \mathrm{J} 1=8.5 \mathrm{~Hz}, \mathrm{~J} 2=2.3 \mathrm{~Hz}), 3.78(\mathrm{~d}, 1 \mathrm{H}, \mathrm{J}=$ $12 \mathrm{~Hz}), 3.47(\mathrm{~d}, 1 \mathrm{H}, \mathrm{J}=12 \mathrm{~Hz}), 3.38(\mathrm{~m}, 1 \mathrm{H}), 3.19(\mathrm{~m}, 1 \mathrm{H}), 2.37(\mathrm{~m}, 2 \mathrm{H}), 2.32(\mathrm{~s}, 3 \mathrm{H}), 2.05(\mathrm{~m}$, 1H), 1.88 (m, 1H), 1.48 (s, 9H). ${ }^{13} \mathrm{C}$ NMR (MeOH-d4, 400 MHz, TMS) $\delta$ 181.26, 164.01, 135.29, $129.79,127.61,127.48,124.17,72.30,57.37,55.70,48.46$, 34.44, 29.56, 28.99, 26.08, 21.26, 19.60. ${ }^{51} \mathrm{~V} \mathrm{NMR}\left(\mathrm{MeOH}-d 4,400 \mathrm{MHz}, \mathrm{VOCl}_{3}\right) \delta-493.95,-465.53, \mathrm{IR}\left(\mathrm{KBr}, \mathrm{cm}^{-1}\right)$ 2956w, 2899w, 2871 w, 2800w, 1640m, 1592s, 1465w, 1442w, 1389m, 1357w, 1301w, 1238s, 1052s, 972s (V=O), 835s, 619vs, 602s. UV-Vis (MeCN; $\left.\lambda_{\max } / \mathrm{nm}\left(\varepsilon / \mathrm{M}^{-1} \mathrm{~cm}^{-1}\right)\right) 258$ (9060), 318 (6040), 352 (6710), 640 (1630). ESI-MS ${ }^{+}:\left[\mathrm{VO}\left(\mathrm{L}^{2}\right)(\mathrm{OMe})+\mathrm{Na}\right]^{+} \mathrm{m} / \mathrm{z}_{\text {calcd. }}=410.114, \mathrm{~m} / \mathrm{z}_{\text {found. }}=410.120 . \mathrm{ESI}-$ $\mathrm{MS}^{-}:\left[\mathrm{V}(\mathrm{O})_{2}\left(\mathrm{~L}^{2}\right)\right]^{-} \mathrm{m} / \mathrm{z}_{\text {calcd. }}=372.102, \mathrm{~m} / \mathrm{z}_{\text {found. }}=372.100$.

[VO(L $\left.\left.{ }^{3}\right)(\mathrm{OMe})(\mathrm{MeOH})\right]$, 3. Yield: $0.32 \mathrm{~g}(83 \%) .{ }^{1} \mathrm{H} \mathrm{NMR}(\mathrm{MeOH}-d 4,400 \mathrm{MHz}, \mathrm{TMS}) \delta 7.29$ (d, $1 \mathrm{H}, J=2.1 \mathrm{~Hz}$ ), $7.14(\mathrm{~d}, 1 \mathrm{H}, J=2.1 \mathrm{~Hz}), 4.06(\mathrm{dd}, 1 \mathrm{H}, J 1=9.1 \mathrm{~Hz}, J 2=2.8 \mathrm{~Hz}), 3.80(\mathrm{~d}, 1 \mathrm{H}, J=$ $12 \mathrm{~Hz}), 3.50(\mathrm{~d}, 1 \mathrm{H}, J=12 \mathrm{~Hz}), 3.25(\mathrm{~m}, 1 \mathrm{H}), 3.17(\mathrm{~m}, 1 \mathrm{H}), 2.39(\mathrm{~m}, 2 \mathrm{H}), 2.36(\mathrm{~s}, 3 \mathrm{H}), 2.05(\mathrm{~m}$, 1H), 1.88 (m, 1H), 1.33 (s, 9H). ${ }^{13} \mathrm{C} \mathrm{NMR}$ (MeOH-d4, $\left.400 \mathrm{MHz}, \mathrm{TMS}\right) \delta$ 181.34, 163.78, 143.76, 127.76, 123.27, 123.07, 122.52, 72.21, 57.02, 55.50, 48.45, 33.58, 30.69, 30.69, 26.01, 21.03, 14,83. ${ }^{51} \mathrm{~V}$ NMR (MeOH-d4, $\left.400 \mathrm{MHz}, \mathrm{VOCl}_{3}\right) \delta-554.19,-494.90,-456.16 . \mathrm{IR}\left(\mathrm{KBr}, \mathrm{cm}^{-1}\right)$ 3168wb, 2950m, 2900w, 1650ms, 1474m, 1345m, 1252m, 1214m, 1050s, 1031s, 973s (V=O), 835m, 777m, 600vs, 552s. UV-Vis (MeCN; $\left.\lambda_{\max } / \mathrm{nm}\left(\varepsilon / \mathrm{M}^{-1} \mathrm{~cm}^{-1}\right)\right) 260$ (8870), 316 (6340), 348 (6180), 635 (1150). ESI-MS $:\left[\mathrm{H}_{2} \mathrm{~L}^{3}+\mathrm{Na}\right]^{+} \mathrm{m} / \mathrm{z}_{\text {calcd. }}=314.173, \mathrm{~m} / \mathrm{z}_{\text {found. }}=314.189$. $\left[\mathrm{VO}\left(\mathrm{L}^{3}\right)(\mathrm{OMe})+\mathrm{Na}\right]^{+} \mathrm{m} / \mathrm{z}_{\text {calcd. }}=410.114, \mathrm{~m} / \mathrm{z}_{\text {found. }}=410.118 . \mathrm{ESI}_{-} \mathrm{MS}^{-}:\left[\mathrm{V}(\mathrm{O})_{2}\left(\mathrm{~L}^{3}\right)\right]^{-} \mathrm{m} / \mathrm{z}_{\text {calcd. }}=$ $372.102, \mathrm{~m} / \mathrm{z}_{\text {found. }}=372.098$.

[VO(L $\left.\left.{ }^{4}\right)(\mathrm{OMe})(\mathrm{MeOH})\right]$, 4. Yield: $0.28 \mathrm{~g}(75 \%) .{ }^{1} \mathrm{H}$ NMR $(\mathrm{MeOH}-d 4,400 \mathrm{MHz}, \mathrm{TMS}) \delta 7.29$ (d, $1 \mathrm{H}, J=2.1 \mathrm{~Hz}), 7.14(\mathrm{~d}, 1 \mathrm{H}, J=2.1 \mathrm{~Hz}), 4.06(\mathrm{dd}, 1 \mathrm{H}, J 1=9.1 \mathrm{~Hz}, J 2=2.8 \mathrm{~Hz}), 3.80(\mathrm{~d}, 1 \mathrm{H}, J=$ $12 \mathrm{~Hz}), 3.50(\mathrm{~d}, 1 \mathrm{H}, J=12 \mathrm{~Hz}), 3.25(\mathrm{~m}, 1 \mathrm{H}), 3.17(\mathrm{~m}, 1 \mathrm{H}), 2.39(\mathrm{~m}, 2 \mathrm{H}), 2.36(\mathrm{~s}, 3 \mathrm{H}), 2.05(\mathrm{~m}$, 1H), 1.88 (m, 1H), 1.33 (s, 9H). ${ }^{13} \mathrm{C} \mathrm{NMR}$ (MeOH-d4, $\left.400 \mathrm{MHz}, \mathrm{TMS}\right) \delta$ 181.34, 163.78, 143.76, $127.76,123.27,123.07,122.52,72.21,57.02$, 55.50, 48.45, 33.58, 30.69, 30.69, 26.01, 21.03, 14.83. ${ }^{51} \mathrm{~V}$ NMR (MeOH-d4, $\left.400 \mathrm{MHz}, \mathrm{VOCl}_{3}\right) \delta-554.19,-494.90,-456.16 . \mathrm{IR}\left(\mathrm{KBr}, \mathrm{cm}^{-1}\right)$ 3168wb, 2950m, 2900w, 1650ms, 1474m, 1345m, 1252m, 1214m, 1050s, 1031s, 973s (V=O), 835m, 777m, 600vs, 552s. UV-Vis (MeCN; $\left.\lambda_{\max } / \mathrm{nm}\left(\varepsilon / \mathrm{M}^{-1} \mathrm{~cm}^{-1}\right)\right) 260$ (8870), 316 (6340), 348 (6180), 635 (1150). ESI-MS $:\left[\mathrm{H}_{2} \mathrm{~L}^{4}+\mathrm{Na}\right]^{+} \mathrm{m} / \mathrm{z}_{\text {calcd. }}=272.126, \mathrm{~m} / \mathrm{z}_{\text {found. }}=272.140$. 


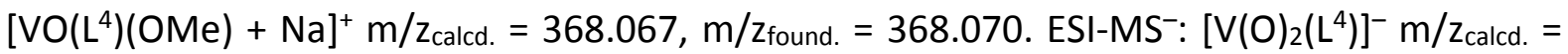
$330.055, \mathrm{~m} / \mathrm{z}_{\text {found. }}=330.076$.

\subsection{NMR spectroscopy}

The ${ }^{1} \mathrm{H},{ }^{13} \mathrm{C}$ and ${ }^{51} \mathrm{~V}$ NMR spectra were recorded with Bruker Avance $400\left({ }^{1} \mathrm{H}: 399.75 \mathrm{MHz},{ }^{13} \mathrm{C}\right.$ : 100.52 MHz, $\left.{ }^{51} \mathrm{~V}: 105.15 \mathrm{MHz}\right)$ and Bruker Avance $500\left({ }^{1} \mathrm{H}: 500.13 \mathrm{MHz},{ }^{13} \mathrm{C}: 125.76 \mathrm{MHz},{ }^{51} \mathrm{~V}\right.$ : 131.55 MHz) NMR spectrometers in $\mathrm{CHCl}_{3}-d$, MeOH-d4, MeCN-d3, DMSO-d6 and PhMe-d8 solutions at $25{ }^{\circ} \mathrm{C}(298 \mathrm{~K})$. The spectrometers were equipped with a broad-band observe probe (Bruker BBO-5 mm-Zgrad). The proton and carbon chemical shifts were referenced to internal TMS (tetramethylsilane; $\delta \mathrm{TMS}=0.00 \mathrm{ppm}$ ). The $0 \mathrm{ppm}$ vanadium reference frequency was calculated from the TMS ${ }^{1} \mathrm{H}$ frequency using the unified chemical shift scale by IUPAC $\left(\equiv\left({ }^{51} \mathrm{~V}, \mathrm{VOCl}_{3}\right)=26.302948\right) \cdot[20]$

The $1 \mathrm{D}{ }^{1} \mathrm{H}$ and ${ }^{51} \mathrm{~V}$ NMR spectra were measured with a single-pulse acquire sequence (flip angles $30^{\circ}$ and $90^{\circ}$ ). The $1 \mathrm{D}^{13} \mathrm{C}$ NMR spectra (flip angle $45^{\circ}$ ) were measured with broad-band ${ }^{1} \mathrm{H}$ decoupling (waltz16). The gradient-selected ${ }^{1} \mathrm{H}\left\{{ }^{1} \mathrm{H}\right\}$ COSY spectra were recorded in doublequantum filtered mode. ${ }^{1} \mathrm{H}\left\{{ }^{1} \mathrm{H}\right\}$ NOE spectra were acquired with the noesygpph pulse sequence using a mixing time of $0.3 \mathrm{~s}$. The ${ }^{1} \mathrm{H}\left\{{ }^{13} \mathrm{C}\right\} \mathrm{HSQC}$ (with multiplicity editing) and $\mathrm{HMBC}$ experiments were optimized for ${ }^{1} \mathrm{~J}_{\mathrm{CH}}$ (short-range) $=145 \mathrm{~Hz}$ and $\mathrm{J}_{\mathrm{CH}}$ (long-range) $=10 \mathrm{~Hz}$. Complete chemical shift assignment for all compounds can be found in the electronic supplementary material.

\subsection{X-ray crystallography}

Single crystal data collection of $\mathbf{1}^{\prime}$ and $\mathbf{4}$ was carried out with an Agilent SuperNova microfocus dual source $(\mathrm{Cu} / \mathrm{Mo})$ diffractometer using CuKa radiation $(\lambda=1.54184 \AA$ ). A suitable crystal was placed in a MiTeGen MicroMount ${ }^{\mathrm{TM}}$ using Fomblin oil and mounted on the diffractometer where it was kept under a stream of liquid nitrogen at $123 \mathrm{~K}$. CrysAlisPro (v. 1.171.37.35 or 1.171.38.41) program[20] was used for data collection and reduction as well as to apply analytical numeric absorption correction based on a multifaceted crystal model.

For 1, the crystallographic data was recorded at $170 \mathrm{~K}$ with a Nonius-Kappa diffractometer equipped with APEXII CCD area-detector using MoKa radiation $(\lambda=0.71073 \AA$ ). Data collection and processing were carried out using COLLECT[21] and DENZO-SMN[22], respectively, 
whereas SADABS[23] was used to apply absorption correction. The structures were solved with either ShelXS by direct methods and refined with ShelXL[24] using Least Squares minimization within the Olex2[25] solution program. All non-hydrogen atoms were refined anisotropically. $\mathrm{C}-\mathrm{H}$ hydrogen atoms were calculated to their ideal positions and refined using a riding model with $U_{\text {iso }}$ values $1.2-1.5$ times to respective host atoms. $\mathrm{O}-\mathrm{H}$ hydrogen atoms were located from the difference map and refined isotropically. CCDC 18551491855152 contain the supplementary crystallographic data for $\mathbf{1}^{\prime} \mathbf{1}^{\prime}, \mathbf{4}$ and $\mathbf{H}_{\mathbf{2}} \mathbf{L}^{\mathbf{1}} \cdot \mathbf{2}$,4-di-tert$\mathrm{BuPhOH}$, respectively.

Table 4. Summary of crystallographic data for $1, \mathbf{1}^{\prime}$ and 4.

\begin{tabular}{|c|c|c|c|}
\hline & 1 & $11^{\prime}$ & 4 \\
\hline Empirical formula & $\mathrm{C}_{22} \mathrm{H}_{36} \mathrm{NO}_{6} \mathrm{~V}$ & $\mathrm{C}_{42.37} \mathrm{H}_{64.37} \mathrm{~N}_{3.19} \mathrm{O}_{10.41} \mathrm{~V}_{2}$ & $\mathrm{C}_{17} \mathrm{H}_{28} \mathrm{NO}_{7} \mathrm{~V}$ \\
\hline Formula weight & 461.46 & 886.77 & 409.34 \\
\hline Temperature/K & 170 & 123 & 123 \\
\hline Crystal system & Monoclinic & Monoclinic & Orthorhombic \\
\hline Space group & $C 2$ & $P 2_{1}$ & $P 2{ }_{1} 2_{1} 2$ \\
\hline$a / \AA ̊$ & $45.4595(7)$ & $16.7775(2)$ & $7.2700(3)$ \\
\hline$b / \AA ̊ A$ & $8.9963(2)$ & $16.60171(17)$ & $28.6337(8)$ \\
\hline$c / \AA ̊ A$ & $12.2069(2)$ & $18.2292(2)$ & $9.3128(2)$ \\
\hline$\alpha /^{\circ}$ & 90 & 90 & \\
\hline$B /{ }^{\circ}$ & $101.9720(10)$ & $115.5289(16)$ & 90 \\
\hline$\gamma / /^{\circ}$ & 90 & 90 & 90 \\
\hline Volume $/ \AA^{3}$ & $4883.64(16)$ & $4581.77(11)$ & $1938.61(10)$ \\
\hline$Z$ & 8 & 4 & 4 \\
\hline$Z^{\prime}$ & 2 & 2 & 1 \\
\hline$\rho_{\text {calc }} \mathrm{g} / \mathrm{cm}^{3}$ & 1.255 & 1.286 & 1.403 \\
\hline$\mu / \mathrm{mm}^{-1}$ & 0.441 & 3.891 & 4.611 \\
\hline$F(000)$ & 1968 & 1881 & 864 \\
\hline Crystal size $/ \mathrm{mm}^{3}$ & $0.38 \times 0.30 \times 0.30$ & $0.221 \times 0.132 \times 0.105$ & $\begin{array}{l}0.211 \times 0.033 \times \\
0.029\end{array}$ \\
\hline Radiation & $\operatorname{MoK} \alpha(\lambda=0.71073)$ & CuK $\alpha(\lambda=1.54184)$ & $\begin{array}{l}\text { CuKa }(\lambda= \\
1.54184)\end{array}$ \\
\hline $2 \Theta$ range for data collection $/^{\circ}$ & 3.41 to 51.996 & 5.992 to 137.966 & 9.496 to 139.958 \\
\hline Index ranges & $\begin{array}{l}-56 \leq h \leq 55,-11 \leq k \\
\leq 10,-14 \leq l \leq 15\end{array}$ & $\begin{array}{l}-20 \leq \mathrm{h} \leq 18,-20 \leq \mathrm{k} \leq \\
15,-19 \leq \mathrm{l} \leq 22\end{array}$ & $\begin{array}{l}-7 \leq h \leq 8,-20 \leq k \\
\leq 34,-11 \leq \mathrm{l} \leq 10\end{array}$ \\
\hline
\end{tabular}




\begin{tabular}{|c|c|c|c|}
\hline Reflections collected & 22910 & 16207 & 5809 \\
\hline Independent reflections & $\begin{array}{l}8754\left[R_{\text {int }}=0.0319,\right. \\
\left.R_{\text {sigma }}=0.0451\right]\end{array}$ & $\begin{array}{l}11698\left[R_{\text {int }}=0.0275,\right. \\
\left.R_{\text {sigma }}=0.0473\right]\end{array}$ & $\begin{array}{l}3637\left[R_{\text {int }}=\right. \\
0.0443, R_{\text {sigma }}= \\
0.0691]\end{array}$ \\
\hline Data/restraints/parameters & $8754 / 75 / 584$ & $11698 / 39 / 1131$ & $3637 / 0 / 248$ \\
\hline Goodness-of-fit on $F^{2}$ & 1.047 & 1.014 & 1.023 \\
\hline Final R indexes $[\mid>=2 \sigma(I)]$ & $\begin{array}{l}R_{1}{ }^{a}=0.0396, w R_{2}{ }^{b}= \\
0.0807^{c}\end{array}$ & $\begin{array}{l}\mathrm{R}_{1}=0.0303, \mathrm{wR}_{2}= \\
0.0718\end{array}$ & $\begin{array}{l}\mathrm{R}_{1}=0.0415, \mathrm{wR}_{2}= \\
0.0970\end{array}$ \\
\hline Final $R$ indexes [all data] & $\begin{array}{l}\mathrm{R}_{1}=0.0460, \mathrm{wR}_{2}= \\
0.0841\end{array}$ & $\begin{array}{l}\mathrm{R}_{1}=0.0331, w \mathrm{R}_{2}= \\
0.0737\end{array}$ & $\begin{array}{l}\mathrm{R}_{1}=0.0471, w \mathrm{R}_{2}= \\
0.1007\end{array}$ \\
\hline Largest diff. peak/hole/ e $\AA^{-3}$ & $0.23 /-0.21$ & $0.21 /-0.28$ & $0.35 /-0.37$ \\
\hline Flack parameter & $0.055(9)$ & $-0.006(3)$ & $0.004(7)$ \\
\hline
\end{tabular}

${ }^{\mathrm{a}} R_{1}=\Sigma|| F_{\mathrm{o}}|-| F_{\mathrm{c}}|| / \Sigma\left|\mathrm{F}_{\mathrm{o}}\right|{ }^{\mathrm{b}} \mathrm{w} R_{2}=\left\{\Sigma\left[\mathrm{w}\left(F_{\mathrm{o}}{ }^{2}-F_{\mathrm{c}}{ }^{2}\right)^{2}\right] / \Sigma\left[\mathrm{w}\left(F_{\mathrm{o}}{ }^{2}\right)^{2}\right]\right\}^{-1 / 2}$ and $\left.\mathrm{w}=1 /\left[\sigma^{2}\left(F_{\mathrm{o}}{ }^{2}\right)+(a P)^{2}+b P\right)\right]$, where $P=\left(2 F_{\mathrm{c}}^{2}-F_{\mathrm{o}}^{2}\right) / 3$. ${ }^{\mathrm{C}}$ Values for reflections with $I>2.0 \sigma(I)$.

\section{Results and discussion}

\subsection{Syntheses}

In the reported literature the ligand precursor $\mathrm{H}_{2} \mathrm{~L}^{1}$ was prepared by one-step Mannich condensation using L-proline, aqueous formaldehyde and 2,4-di-tert-butylphenol in a 1:4:2 ratio and allowing the reaction mixture to reflux for two days.[19] However, our attempts at following this procedure led to the formation of impure products, which were difficult to purify via crystallization. For example, we were able to isolate $\mathrm{H}_{2} \mathrm{~L}^{1}$ by crystallization from acetonitrile reaction medium as 1:1 adduct with unreacted 2,4-di-tert-butylphenol. Therefore, the proligands used in this study were prepared by refluxing L-proline, aqueous formaldehyde and appropriate 2,4-disubstituted phenols in a 1:1:1 ratio in methanol overnight, and purified via column chromatography with yields of 60-94\%. Refer to the electronic supplementary material for detailed syntheses and purification of the proligands.

To obtain the corresponding oxidovanadium(v) complexes, the ligand precursors $\mathrm{H}_{2} \mathrm{~L}^{1-4}$ were allowed to react in methanol with various vanadium(IV/V) precursors, i.e. $\mathrm{VO}(\mathrm{acac})_{2}, \mathrm{VOSO}_{4}$. $5 \mathrm{H}_{2} \mathrm{O}$ and $\mathrm{VO}(\mathrm{OPr})_{3}$ (Scheme 1). Regardless of used vanadium starting material, the same oxidovanadium( $\mathrm{V})$ complex of the general formula $\left[\mathrm{VO}\left(\mathrm{L}^{1-4}\right)(\mathrm{OMe})(\mathrm{MeOH})\right]$ could be isolated as brown crystals with yields of ca. $30-80 \%$. The reactions with $\mathrm{VO}(\mathrm{acac})_{2}$ were often slow and incomplete. Conversely, brownish red compounds $\mathbf{1 - 4}$ were obtained in nearly 
quantitative yields by stirring the mixture of the proligands and $\mathrm{VO}(\mathrm{OPr})_{3}$ in methanol at 60 ${ }^{\circ} \mathrm{C}$ for two hours followed by crystallization at $-25^{\circ} \mathrm{C}$. However, to avoid use of the moisturesensitive alkoxide precursor, the complexes were eventually prepared using $\mathrm{VOSO}_{4} \cdot 5 \mathrm{H}_{2} \mathrm{O}$ in warm methanol in the presence of triethylamine under ambient atmosphere. The reactions involving vanadium(IV) precursors were done under ambient atmosphere to facilitate oxidation of $\mathrm{V}(\mathrm{IV})$ to $\mathrm{V}(\mathrm{V})$. All complexes are stable in dry solvents for several days but hydrolyse slowly in the presence of trace amounts of water. For example, prolonged storing of complex 1 in moist acetonitrile lead to the formation and deposition of dinuclear $\left[\left\{\mathrm{VO}\left(\mathrm{L}^{1}\right)\right\}_{2}(\mu-\mathrm{O})\right] \cdot \mathrm{H}_{2} \mathrm{O}\left(\mathbf{1}^{\prime}\right)$ as black crystals (see the electronic supplementary material for the $\mathrm{x}$-ray structure). It is well known that oxidovanadium(v) aminobisphenolates can form related dinuclear species in acetonitrile solutions. $[6,9,12,26,27]$

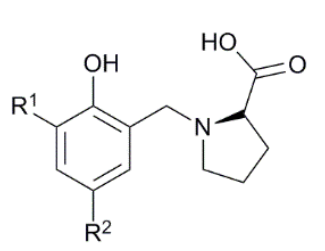

$\mathrm{H}_{2} \mathrm{~L}^{1}: \mathrm{R}^{1}=\mathrm{R}^{2}=\mathrm{t}-\mathrm{Bu}$

$\mathrm{H}_{2} \mathrm{~L}^{2}: \mathrm{R}^{1}=\mathrm{t}-\mathrm{Bu}, \mathrm{R}^{2}=\mathrm{Me}$

$\mathrm{H}_{2} \mathrm{~L}^{3}: \mathrm{R}^{1}=\mathrm{Me}, \mathrm{R}^{2}=\mathrm{t}-\mathrm{Bu}$ $\mathrm{H}_{2} \mathrm{~L}^{4}: \mathrm{R}^{1}=\mathrm{R}^{2}=\mathrm{Me}$
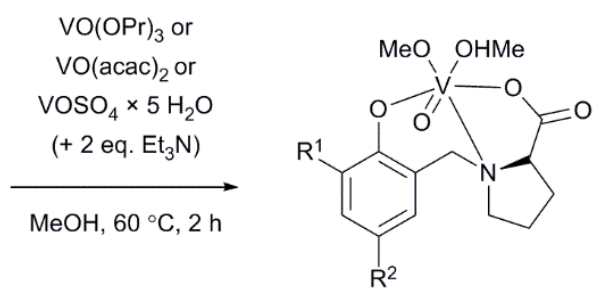

$1-4$

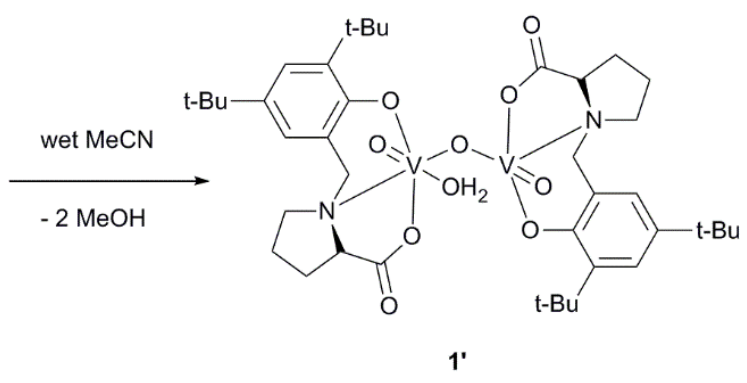

Scheme 1. Formation of the complexes $\mathbf{1 - 4}$ in methanol and subsequent hydrolysis of $\mathbf{1}$ to form dinuclear $\mathbf{1}^{\prime}$ in acetonitrile in the presence of trace amounts of water.

\subsection{Spectroscopic characterization and molecular structures}

The structures of all compounds were verified by IR, UV-Vis and CD (circular dichroism), ${ }^{1} \mathrm{H}$, ${ }^{13} \mathrm{C}$ and ${ }^{51} \mathrm{~V}$ NMR spectroscopy. Furthermore, ESI-TOF high-resolution mass spectrometry was used to study the structures of the complexes, whereas single crystal x-ray diffraction was used to determine the solid state structures of $\mathbf{1 , 4}$ and $\mathbf{1}^{\prime}$.

\subsection{IR spectroscopy}

The complete IR spectral data is given in the electronic supplementary information. The spectra of all proligands and complexes are, largely, identical to one another, as the only changes in the structures are the alkyl groups in the aromatic ring. The most prominent feature seen in the spectra of the proligands is a very broad absorption at ca. $2500-3500 \mathrm{~cm}^{-}$ 
${ }^{1}$, corresponding to phenolic and carboxylic $\mathrm{OH}$ groups. Accordingly, this signal is missing in the IR spectra of the complexes, indicating that the ligands are coordinated to the metal centre via phenolate and carboxylate oxygens. All the other signals in the spectra of the proligands are also present in the spectra of the complexes, with the exception of the $V=0$ stretching vibration at $972-976 \mathrm{~cm}^{-1}$ in all complexes. The experimental values agree well with other oxidovanadium complexes with an ONO donor set,[6,9,11] as well as with theoretical values obtained in other studies.[28]

\subsection{UV-Vis and CD spectroscopy}

The UV-Vis and CD spectra of all proligands $\left(1.00 \times 10^{-4} \mathrm{M}\right)$ and complexes $\left(6.70 \times 10^{-5} \mathrm{M}\right)$ were measured in acetonitrile. Complete list of the spectral data can be found in the electronic supplementary material. The UV-Vis spectra of the proligands have low intensity absorption bands $\left(\varepsilon \approx 1,400 \mathrm{M}^{-1} \mathrm{~cm}^{-1}\right)$ at ca. $285 \mathrm{~nm}$, which are assigned to the $\left(\mathrm{n} \rightarrow \pi^{*}\right) \mathrm{CT}$ band of the aromatic ring system. The most intensive $\left(\varepsilon \sim 7,500 \mathrm{M}^{-1} \mathrm{~cm}^{-1}\right)$ primary absorption band is assigned to several merged signals, namely the $\left(\pi \rightarrow \pi^{*}\right)$ transitions of the aromatic ring system and the $\left(\pi \rightarrow \pi^{*}\right)$ and $\left(n \rightarrow \pi^{*}\right)$ transitions of the carbonyl group. These transitions are also present in the complexes, although the signals have red shifted ca. $100 \mathrm{~nm}$ and are more intensive. For the complexes, a broad low intensity band (avg. $\varepsilon \sim 1,400 \mathrm{M}^{-1} \mathrm{~cm}^{-1}$ ) not displayed by the ligands can be observed at ca. $640 \mathrm{~nm}$. For all complexes the metal centres are in their highest oxidation state i.e. vanadium(V), which is a $d^{0}$ species. Thus, with no $d-$ electrons, $d-d$ transitions cannot be observed. As such, the low intensity band must be assigned as ligand to metal charge transfer (LMCT) transitions from the phenolate to the vanadium empty d-orbital $(\pi \rightarrow \mathrm{d})$.[29] These transitions are common in vanadium(V) compounds bearing an aminebisphenol ligand[12], but can be extended to concern vanadium(v) complexes with only one phenolate moiety.

\subsection{NMR spectroscopy}

The structures of the organic proligands and the complexes in solution were studied by ${ }^{1} \mathrm{H}$, ${ }^{13} \mathrm{C}$ and additionally, for complexes, by ${ }^{51} \mathrm{~V}$ NMR. The structures could be adequately assessed, and verified to be correct, by $1 \mathrm{D}{ }^{1} \mathrm{H}$ and ${ }^{13} \mathrm{C}$ NMR studies. However, the aliphatic $\mathrm{CH}_{2}$ protons in the 5-membered proline moiety of the ligands have very complicated splitting of the 
resonances. This is to be expected as the chirality of the proligands imparts magnetic nonequivalence across all protons, a matter that is further complicated by coupling to neighbouring (inequivalent) protons. Consequently, appropriate 2D NMR experiments (DQF-COSY, HMBC, HSQC and NOESY) were undertaken to fully understand the structures of the ligands and the complexes. Due to the similar nature of all the compounds, $\mathbf{4}$ was chosen as a representative for all of the other complexes, and its structure was thoroughly investigated. For the available 2D spectra in methanol- $d 4$ for 4 , together with interpretations, see S29S37.

The structure elucidation of $\mathbf{4}$ was separated into aromatic and aliphatic (proline moiety) parts. In the aromatic moiety, the two protonated carbons i.e. $\mathrm{C}^{3}$ and $\mathrm{C}^{5}$ were identified by HSQC, HMBC and NOESY. The chemical shift of $\mathrm{H}^{3}$ was established at $7.06 \mathrm{ppm}$ through NOESY as it has a spatial NOE correlation with both ortho and para methyl group protons e.g. $\mathrm{H}^{13}$ and $\mathrm{H}^{14}$, respectively. Accordingly, $\mathrm{H}^{5}$ has only one NOE correlation with the adjacent methyl protons $\mathrm{H}^{14}$. Carbons $\mathrm{C}^{3}, \mathrm{C}^{5}, \mathrm{C}^{13}$ and $\mathrm{C}^{14}$ were then appropriately assigned via $\mathrm{HSQC}$. HMBC measurements supported the previous assignations and were further used to identify benzylic $\mathrm{C}^{7}$ methylene bridge (and $\mathrm{H}^{7 \mathrm{~A} / \mathrm{B}}$ via $\mathrm{HSQC}$ ), and $\mathrm{C}^{1}$.

In the proline moiety of $4, \mathrm{CH}_{2}$ edited HSQC was employed to establish $\mathrm{C}^{11} \rightarrow \mathrm{H}^{11}$ correlation as it is the only positive $\mathrm{CH}$-type correlation found. $\mathrm{H}^{11}$ COSY and HSQC experiments were used to assign $\mathrm{C}^{8}-\mathrm{C}^{10}$ and $\mathrm{H}^{8 \mathrm{~A} / \mathrm{B}}-\mathrm{H}^{10 \mathrm{~A} / \mathrm{B}}$. See the Supplementary Information for full list of ${ }^{1} \mathrm{H}$ and ${ }^{13} \mathrm{C}$ NMR data. The data obtained from the 2D measurements (for 4) was used to assign the ${ }^{1} \mathrm{H}$ and ${ }^{13} \mathrm{C}$ NMR signals in $\mathbf{2}-4$ and $\mathrm{H}_{2} \mathrm{~L}^{1-4}$ as well, because the spectra are closely similar to one another.

The structure dynamics of the complexes in solution were further studied using ${ }^{51} \mathrm{~V} \mathrm{NMR}$, using complex 1 as the reference. Interestingly, the structure of 1 was found to be strongly dependent on the used NMR solvent (ESI figure S28). ${ }^{51} \mathrm{~V}$ NMR measurements at room temperature in $\mathrm{CDCl}_{3}$ and toluene- $d 8$ indicate that three compounds are present at $-480-$ -550 ppm with ca. 2:1:1 ratios in $\mathrm{CDCl}_{3}$, and 2:2:1 in toluene, respectively. In DMSO- $d 6$, there are three broad, partially overlapping signals at $-450--500 \mathrm{ppm}$. The most complicated spectrum is obtained in acetonitrile wherein as many as five major resonances as well as several minor ones are observed. Remarkably, when measured in $\mathrm{MeOH}-d 4$ both the ${ }^{1} \mathrm{H}$ and 
${ }^{51} \mathrm{~V}$ spectra indicate the presence of only one major component, with $\geq 95 \%$ dominance over a minor component. It can be reasonably assumed that the signals represent vanadiumcontaining structures having varying coordination number(s) and/or coordinated solvent molecules. The unambiguous determination of the exact nature of the structures observed in the different solvents is, however, rather challenging; the structures are transient, and unisolable via basic separation methods such as column chromatography.

Dynamic solution behaviour, as evidenced by ${ }^{51} \mathrm{~V} N M R$, for oxidovanadium( $\mathrm{V}$ ) complexes bearing related aminophenolate ligand systems is well known. $[6,8,9,11,12,30-32]$ An oxidomethoxidovanadium(v) complex bearing tricoordinatively bonded potentially tetradentate aminobisphenolate ligand, which is structurally very similar to $\mathbf{1}-\mathbf{4}$, has multiple signals observed in ${ }^{51} \mathrm{~V}$ NMR.[6] These signals predominantly arise from coordination isomers involving axial and equatorial ligands changing coordination sites, as well as from potential dimers. Owing to the stark similarities between these oxidovanadium(v) systems and ours, we have made a tentative suggestion that the observed multiple ${ }^{51} \mathrm{~V} N M R$ signals are the result of similar rapid exchange of ligands, and/ or different conformers. The matter is further substantiated by the fact that our ligands are tridentate, providing a less rigid framework for the oxidovanadium(v) center compared to tetradentate ligands, by virtue of allowing two coordination sites for labile ligands. Additionally, some ${ }^{51} \mathrm{~V}$ NMR signals may originate from dimerized oxidovanadium(v) complexes, given that we were able to obtain $\mathbf{1}^{\prime}$ from acetonitrile. Dimeric species, although not always encountered, are known to form with many oxidovanadium(v) systems. $[6,9,12,26,27]$

\subsection{Crystal and molecular structures}


Complexes $\mathbf{1}$ and $\mathbf{4}$ were recrystallized from methanol as dark brown needles and their solid state molecular structures were verified by single crystal X-ray diffraction studies. They both crystallize in non-centrosymmetric space groups owing to the chiral carbon in the L-proline moiety. 1 crystallizes in a monoclinic space group $C 2$ and has two chemically identical, although crystallographically unique molecules in the asymmetric unit (1A and $\mathbf{1 B}$ in Table $\mathbf{1})$ with one of the two complex units showing a two-fold disorder of the pyrrolidine ring. 4 crystallizes in an orthorhombic space group $P 2{ }_{1} 2{ }_{1} 2$ with molecules of non-coordinated methanol in the crystal lattice. The compounds are analogous mononuclear complexes in which the respective amino acid phenols are coordinated to the oxidovanadium(V) ion as dianionic tridentate ligands. The distorted octahedral coordination sphere is completed by a methoxide group, which is coordinated trans to the proline nitrogen donor, and a methanol molecule which lies trans to the oxo group (see Figure 2).
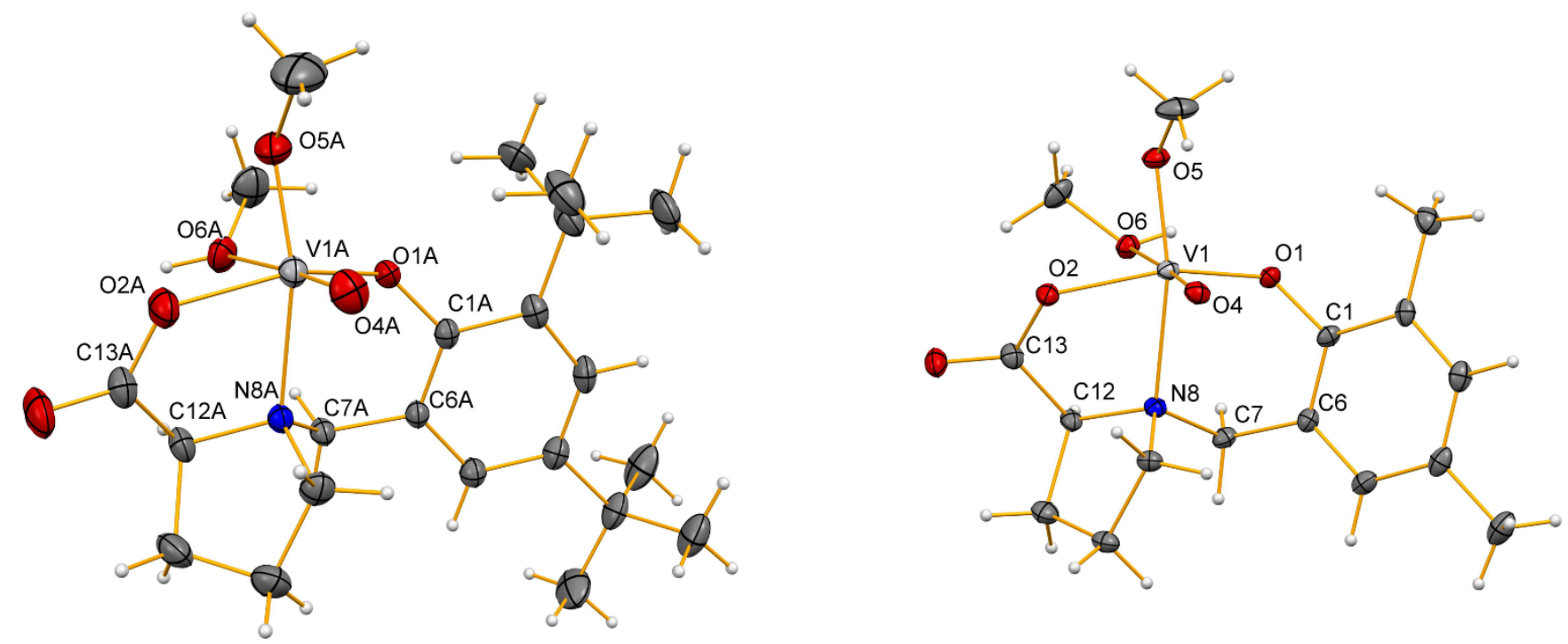

Figure 2. The molecular structure of $\mathbf{1}$ (left) and $\mathbf{4}$ (right). Only one of the two molecules in the asymmetric unit of $\mathbf{1}$ is shown. Thermal ellipsoids are presented at the $30 \%$ level of probability.

The two complexes show very similar bonding parameters in respect to the coordination environment of the metal (see Table 1 for structure specific bond parameters). The $V=0$ distances are $1.58-1.60 \AA$, whereas the $\mathrm{V}-\mathrm{O}_{\text {phenolate }}$ and $\mathrm{V}-\mathrm{O}_{\text {carboxylate }}$ distances lie between $1.82-1.85 \AA$ and $1.96-1.97 \AA$, respectively. The deprotonated methoxide group and neutral methanol molecule are bonded to the metal ion with the respective $\mathrm{V}-\mathrm{O}$ methoxide and $\mathrm{V}-$ 


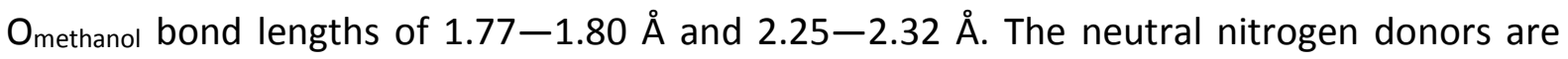
coordinated to vanadium ions with the bond lengths of $2.23-2.25 \AA$. A visual comparison of the crystal structures of $\mathbf{1}$ and $\mathbf{4}$ is shown in figure S45, which further demonstrates the general geometrical similarity of the complexes. The most apparent geometrical differences arise from the orientation of the methoxide and methanol ligands that have significant rotational freedom with respect to the $\mathrm{V}-\mathrm{O}$ bond axis.

In addition to 1 we were able to analyse the related dinuclear complex $\mathbf{1}^{\prime}$ by means of single crystal X-ray diffraction. 1' crystallizes in the space group $P 2{ }_{1}$ and consists of two independent molecules in the asymmetric unit (see S44 in Supplementary Information). The structure has both acetonitrile and water solvent molecules included in the crystal lattice with respective amounts of 1.2 and 0.4 molecules per each complex formula unit. The molecular structure of the complex is formed of two different V-centred units. In one unit, the metal ion has an approximate square pyramidal coordination sphere in which the central atom is coordinated to the tridentate amino acid phenolate ligand as well as to one terminal and one bridging oxido ligands. The other unit in the dinuclear molecule has a hexacoordinated vanadium centre with a coordination sphere resembling the structure of 1 with the bridging oxido and a water molecule completing the coordination environment instead of the methoxide and methanol ligands found in 1.

Table 1. Selected bond angles $\left({ }^{\circ}\right)$ and distances $(\AA)$ in complexes $\mathbf{1}$ and $\mathbf{4}$.

\begin{tabular}{|l|l|l|l|}
\hline \multicolumn{5}{|l|}{} & 1A & 1B & \multicolumn{1}{l|}{} \\
\hline \multicolumn{5}{|l|}{ Distances } \\
\hline V1-O1 & $1.838(2)$ & $1.821(2)$ & $1.847(3)$ \\
\hline V1-O2 & $1.963(3)$ & $1.972(3)$ & $1.964(3)$ \\
\hline V1-O2 & $1.963(3)$ & $1.972(3)$ & $1.964(3)$ \\
\hline V1-O2 & $1.963(3)$ & $1.972(3)$ & $1.964(3)$ \\
\hline V1-O4 & $1.587(3)$ & $1.576(3)$ & $1.597(3)$ \\
\hline V1-O5 & $1.775(3)$ & $1.775(3)$ & $1.800(3)$ \\
\hline V1-O5 & $2.282(3)$ & $2.324(3)$ & $2.253(3)$ \\
\hline V1-N8 & $2.232(3)$ & $2.250(3)$ & $2.250(3)$ \\
\hline \multicolumn{5}{|c|}{ Angles } & $151.30(13)$ \\
\hline O1-V1-O2 & $154.67(13)$ & $153.33(11)$ & $166.01(13)$ \\
\hline O5-V1-N8 & $162.77(12)$ & $161.36(13)$ & $176.48(14)$ \\
\hline O4-V1-06 & $176.99(15)$ & $175.03(15)$ & $91.22(13)$ \\
\hline O4-V1-N8 & $94.96(13)$ & $93.39(14)$ & \\
\hline
\end{tabular}




\section{Conclusions}

Chiral, tridentate amino acid phenol ligand precursors $\mathrm{H}_{2} \mathrm{~L}^{1-4}$ can be easily prepared from $L$ proline, 2,4-disubstituted phenols and formaldehyde in a one-pot fashion. These tridentate proligands react with oxidovanadium precursors $\mathrm{VO}(\mathrm{acac})_{2}, \mathrm{VOSO}_{4} \cdot 5 \mathrm{H}_{2} \mathrm{O}$ and $\mathrm{VO}(\mathrm{OPr})_{3}$ in methanol to form oxidomethoxidovanadium $(\mathrm{V})$ complexes of the general formula of [VO( $\mathrm{L}^{1-}$ $\left.\left.{ }^{4}\right)(\mathrm{OMe})(\mathrm{MeOH})\right]$. The $x$-ray studies show that the complexes are octahedrally coordinated in the solid state, having structures resembling that found in the active site of VBrPO. However, in solution the NMR spectra indicate the presence of several different conformations or isomeric structures, depending on the solvent. This fact significantly hampers any further solution state studies, including catalytic experiments, as it would be difficult to draw any meaningful structure-reactivity conclusions.

\section{Notes}

The authors declare no competing interests.

\section{Acknowledgements}

A.P. gratefully acknowledges the financial support from the Academy of Finland (project no. 315911).

\section{References}

[1] D.C. Crans, J.J. Smee, E. Gaidamauskas, L. Yang, The Chemistry and Biochemistry of Vanadium and the Biological Activities Exerted by Vanadium Compounds, Chem. Rev. 104 (2004) 849902. doi:10.1021/cr020607t.

[2] C. Bolm, Vanadium-catalyzed asymmetric oxidations, Coord. Chem. Rev. 237 (2003) 245-256. doi:10.1016/S0010-8545(02)00249-7.

[3] M. Kirihara, Aerobic oxidation of organic compounds catalyzed by vanadium compounds, Coord. Chem. Rev. 255 (2011) 2281-2302. doi:10.1016/j.ccr.2011.04.001.

[4] D. Rehder, The medicinal and catalytic potential of model complexes of vanadate-dependent haloperoxidases, Coord. Chem. Rev. 237 (2003) 53-63. doi:10.1016/S0010-8545(02)00300-4.

[5] A.A. El-Taras, I.M. EL-Mehasseb, A.E.-M.M. Ramadan, Synthesis, characterization, magnetic, thermal and electrochemical studies of oxidovanadium (IV) picolyl hydrazones as functional catechol oxidase models, Comptes Rendus Chim. 15 (2012) 298-310. doi:10.1016/j.crci.2011.11.009.

[6] M.R. Maurya, B. Uprety, F. Avecilla, P. Adão, J. Costa Pessoa, Vanadium( $\langle\operatorname{scp}\rangle \mathrm{v}</ \operatorname{scp}\rangle$ ) complexes of a tripodal ligand, their characterisation and biological implications, Dalt. Trans. 44 (2015) 17736-17755. doi:10.1039/C5DT02716K. 
[7] S.K. Mal, M. Mitra, H.R. Yadav, C.S. Purohit, A.R. Choudhury, R. Ghosh, Synthesis, crystal structure and catecholase activity of a vanadium(V) Schiff base complex, Polyhedron. 111 (2016) 118-122. doi:10.1016/j.poly.2016.03.033.

[8] P. Salonen, A. Peuronen, A. Lehtonen, Oxidovanadium(V) amine bisphenolates as epoxidation, sulfoxidation and catechol oxidation catalysts, Inorg. Chem. Commun. 86 (2017) 165-167. doi:10.1016/j.inoche.2017.10.017.

[9] M.R. Maurya, B. Uprety, F. Avecilla, P. Adão, M.L. Kuznetsov, J. Costa Pessoa, Solution Behaviour and Catalytic Potential towards Oxidation of Dopamine by Oxidovanadium(V) Complexes of Tripodal Tetradentate Ligands, Eur. J. Inorg. Chem. 2017 (2017) 3087-3099. doi:10.1002/ejic.201700342.

[10] M.R. Maurya, M. Bisht, A. Kumar, M.L. Kuznetsov, F. Avecilla, J.C. Pessoa, Synthesis, characterization, reactivity and catalytic activity of oxidovanadium(IV), oxidovanadium(V) and dioxidovanadium(V) complexes of benzimidazole modified ligands, Dalt. Trans. 40 (2011) 6968-6983. doi:10.1039/c1dt10261c.

[11] M.K. Hossain, M. Haukka, G.C. Lisensky, A. Lehtonen, E. Nordlander, Oxovanadium(V) complexes with tripodal bisphenolate and monophenolate ligands: Syntheses, structures and catalytic activities, Inorganica Chim. Acta. 487 (2019) 112-119. doi:10.1016/j.ica.2018.11.049.

[12] S. Barroso, P. Adão, F. Madeira, M.T. Duarte, J.C. Pessoa, A.M. Martins, Vanadium diaminebis(phenolate) complexes: Syntheses, structures, and reactivity in sulfoxidation catalysis, Inorg. Chem. 49 (2010) 7452-7463. doi:10.1021/ic1007704.

[13] B. Floris, F. Sabuzi, A. Coletti, V. Conte, Sustainable vanadium-catalyzed oxidation of organic substrates with $\mathrm{H} 2 \mathrm{O}$ 2, Catal. Today. 285 (2017) 49-56. doi:10.1016/j.cattod.2016.11.006.

[14] S. Groysman, I. Goldberg, Z. Goldschmidt, M. Kol, Vanadium(III) and vanadium(V) amine tris(phenolate) complexes, Inorg. Chem. 44 (2005) 5073-5080. doi:10.1021/ic050084i.

[15] M. Mba, M. Pontini, S. Lovat, C. Zonta, G. Bernardinelli, P.E. Kündig, G. Licini, C3 vanadium(V) amine triphenolate complexes: Vanadium haloperoxidase structural and functional models, Inorg. Chem. 47 (2008) 8616-8618. doi:10.1021/ic8015113.

[16] O. Wichmann, R. Sillanpää, A. Lehtonen, Structural properties and applications of multidentate [O,N,O, $\left.X^{\prime}\right]$ aminobisphenolate metal complexes, Coord. Chem. Rev. 256 (2012) 371-392. doi:10.1016/j.ccr.2011.09.007.

[17] M. Weyand, H.-J. Hecht, M. Kieß, M.-F. Liaud, H. Vilter, D. Schomburg, X-ray structure determination of a vanadium-dependent haloperoxidase from Ascophyllum nodosum at $2.0 \AA$ resolution 1 1Edited by R. Huber, J. Mol. Biol. 293 (1999) 595-611. doi:10.1006/jmbi.1999.3179.

[18] U. Christmann, H. Dau, M. Haumann, E. Kiss, P. Liebisch, D. Rehder, G. Santoni, C. Schulzke, Substrate binding to vanadate-dependent bromoperoxidase from Ascophyllum nodosum: A vanadium K-edge XAS approach, Dalt. Trans. 0 (2004) 2534-2540. doi:10.1039/b405764c.

[19] H. Sheykhi, E. Safaei, A proline-based aminophenol ligand: Synthesis, iron complexation, magnetic, electronic and redox investigation, Spectrochim. Acta Part A Mol. Biomol. Spectrosc. 118 (2014) 915-920. doi:10.1016/j.saa.2013.09.003.

[20] R.K. Harris, E.D. Becker, S.M. Cabral de Menezes, R. Goodfellow, P. Granger, NMR nomenclature: nuclear spin properties and conventions for chemical shifts. IUPAC 
Recommendations 2001. International Union of Pure and Applied Chemistry. Physical Chemistry Division. Commission on Molecular Structure and Spectroscopy, Magn. Reson. Chem. 40 (2002) 489-505. doi:10.1002/mrc.1042.

[21] Nonius (1998), COLLECT, Nonius BV, Delft, The Netherlands, n.d.

[22] Z. Otwinowski, M. Minor, Methods in Enzymology, in: C.W.J. Carter, R.M. Sweet (Eds.), Macromol. Crystallogr. Part A, New York, USA, 1997: pp. 307-326.

[23] Bruker (2001), SADABS, Bruker ACS Inc., Madison, Wisconsin, USA, n.d.

[24] G.M. Sheldrick, Crystal structure refinement with SHELXL, Acta Crystallogr. Sect. C Struct. Chem. 71 (2015) 3-8. doi:10.1107/S2053229614024218.

[25] O. V Dolomanov, L.J. Bourhis, R.J. Gildea, J.A.K. Howard, H. Puschmann, OLEX2 : a complete structure solution, refinement and analysis program, J. Appl. Crystallogr. 42 (2009) 339-341. doi:10.1107/S0021889808042726.

[26] M. Debnath, M. Dolai, K. Pal, S. Bhunya, A. Paul, H.M. Lee, M. Ali, Mono- and dinuclear oxidovanadium( $\langle s c p\rangle v</ s c p\rangle$ ) complexes of an amine-bis(phenolate) ligand with bromoperoxidase activities: synthesis, characterization, catalytic, kinetic and computational studies, Dalt. Trans. 47 (2018) 2799-2809. doi:10.1039/C7DT04718E.

[27] A.I. Elkurtehi, A.G. Walsh, L.N. Dawe, F.M. Kerton, Vanadium Aminophenolate Complexes and Their Catalytic Activity in Aerobic and H2O2-Mediated Oxidation Reactions, Eur. J. Inorg. Chem. 2016 (2016) 3123-3130. doi:10.1002/ejic.201600068.

[28] J. Costa Pessoa, M.J. Calhorda, I. Cavaco, I. Correia, M.T. Duarte, V. Felix, R.T. Henriques, M.F.M. Piedade, I. Tomaz, Molecular modelling studies of $\mathrm{N}$-salicylideneamino acidato complexes of oxovanadium(iv). Molecular and crystal structure of a new dinuclear LOVIV-OVVOL mixed valence complex, J. Chem. Soc. Dalt. Trans. (2002) 4407. doi:10.1039/b205843j.

[29] J.A. Bonadies, C.J. Carrano, Vanadium phenolates as models for vanadium in biological systems. 1. Synthesis, spectroscopy, and electrochemistry of vanadium complexes of ethylenebis[(o-hydroxyphenyl)glycine] and its derivatives, J. Am. Chem. Soc. 108 (1986) 4088-4095. doi:10.1021/ja00274a038.

[30] J.-B. Wang, L.-P. Lu, J.-Y. Liu, H.-L. Mu, Y.-S. Li, [ONNO]-type oxovanadium(V) complexes containing amine pyridine bis(phenolate) ligands: synthesis, characterization and catalytic behavior for ethylene (co)polymerization, J. Mol. Catal. A Chem. 398 (2015) 289-296. doi:10.1016/j.molcata.2014.12.016.

[31] F. Wolff, C. Lorber, R. Choukroun, B. Donnadieu, Vanadium(V) Complexes of a Chelating Dianionic [ONNO]-Type Amine Bis(Phenolate) Ligand: Synthesis and Solid State and Solution Structures, Inorg. Chem. 42 (2003) 7839-7845. doi:10.1021/ic034742f.

[32] M.M. Hänninen, A. Peuronen, P. Damlin, V. Tyystjärvi, H. Kivelä, A. Lehtonen, Vanadium complexes with multidentate amine bisphenols, Dalt. Trans. 43 (2014) 14022-14028. doi:10.1039/C4DT01007H. 